\title{
The effect of exercise on reelin level in the hippocampus of diabetic rats
}

\author{
Khoshghadam $\mathrm{S}^{1}$, Sheikhzadeh Hesari $\mathrm{F}^{1}$, Ahmadiasl $\mathrm{N}^{2}$, Dastranj Tabrizi A $\mathrm{A}^{3}$, \\ Hosseinzadeh $\mathrm{SS}^{3}$, Ramouz $\mathrm{A}^{4}$
}

Animal study group, Faculty of Natural Sciences, University of Tabriz, Tabriz, Iran.

fsheikhzadeh@tabrizu.ac.ir

\begin{abstract}
AIM: Diabetes is one of the most common diseases which can attenuate brain function by destroying hippocampus neurons, while reelin is a largely secreted extracellular matrix glycoprotein in the hippocampus causing synaptic plasticity, promoting postsynaptic structures and maturing neurons. The aim of this study was to assess the effect of exercise, as an external factor for neurogenesis in the brain, on reelin levels and memory improvement in diabetic rats.

METHOD: Thirty rats were randomly allocated into three groups; healthy sedentary, diabetic sedentary and diabetic exercise-trained. The experimental group was treadmill-exercised at speed $22 \mathrm{~m} / \mathrm{min}$ for 1 hour, 5 days per week. Finally, spatial memory of rats was tested and reelin levels were measured.

RESULTS: The results showed that short-term exercise improved spatial memory in diabetic rats but had no effect on reelin levels in the hippocampus of diabetic rats.

CONCLUSION: Diabetes reduced the spatial memory without altering the reelin levels while exercise improved spatial memory without altering the reelin levels (Fig. 4, Ref. 33). Text in PDF www.elis.sk. KEY WORDS: reelin, exercise, diabetes, memory, hippocampus.
\end{abstract}

\section{Introduction}

Type 1 diabetes, also known as insulin-dependent diabetes, occurs due to disturbance in sugar (glucose) metabolism. The lack of insulin secretion by the pancreas gland is the main underlying reason for type 1 diabetes (1). Several metabolic disorders such as diabetes mellitus (DM) may contrive molecular and cellular changes in central nervous system, especially in the hippocampus (2). Diabetes significantly reduces neuronal density in the dentate gyrus of hippocampus (3), which plays a crucial role in proper processing and spatial memory (4). On the other hand, learning and memory defects are more prevalent among diabetics compared to non-diabetic individuals (5). Type 1 diabetes leads to impaired learning, memory and cognition in animals

\begin{tabular}{l}
\hline${ }^{1}$ Animal Study Group, Faculty of Natural Sciences, University of Tabriz, \\
Tabriz, Iran, ${ }^{2}$ Department of Physiology, Faculty of Medicine, Tabriz Uni- \\
versity of Medical Sciences, Tabriz, Iran, ${ }^{3}$ Department of Pathology, Fac- \\
ulty of Medicine, Tabriz University of Medical Sciences, Tabriz, Iran, \\
and ${ }^{4}$ General, Visceral and Transplant Surgery Department, Universitat \\
Klinikum Heidelberg, Heidelberg, Germany
\end{tabular}

Address for correspondence: F.S. Hesari, Animal Study Group, Faculty of Natural Sciences, University of Tabriz, Daneshgah Street, Tabriz, East Azerbaijan, Iran. Postal code: 51666-16471.

Phone: +989141170587

Acknowledgement: This work was financially supported by Neurosciences Research Center, Tabriz University of Medical Sciences, Tabriz, Iran and Faculty of Natural Sciences, University of Tabriz, Tabriz, Iran.
(6) and increases the risk of dementia, Alzheimer's disease and schizophrenia $(7,8)$.

Reelin is an extracellular glycoprotein that binds to the transmembrane receptors through APOE2 and VLDLR and activates Src tyrosine kinase. This cascade of enzymes is essential for long-term potentiation (LTP) and stimulation of NMDA receptors that play important roles in learning, memory, recalling information, and creating long-term potentiation (9). Reelin is necessary for proper development of the brain during the embryonic period and early organization of the cerebral cortex and cerebellum, as well as decent neuronal migration and radial glial cells migration into the suitable position (10). Reelin has an impact on the release of neurotransmitters in an adult brain, long-term memory formation and synaptic plasticity (11). This glycoprotein is generated by the hippocampus and cerebellum and is expressed for the whole life in adult brain (12).

It is well understood that neurogenesis is not a static process, it can be influenced by both external and internal factors and occurs in all parts of fetal brain and only in a limited area of adult brain including the hippocampus (13). On this basis, physical activity is an external factor that reduces blood glucose levels, increases cognitive performance and stimulates neurogenesis in the dentate gyrus of hippocampus $(14,15)$.

In general, there is much evidence about the relationship between exercise, neurogenesis, memory and mental function improvement in diabetic patients. Although, many studies have investigated the effect of reelin on neurogenesis in the hippocampus, 


\section{7-110}

the aim of this study was to evaluate the effect of exercise on reelin levels in hippocampus and its effects on diabetes' complications, including spatial memory status.

\section{Materials and methods}

\section{Animals}

Thirty adult male Wistar rats weighing $250 \pm 50 \mathrm{~g}$, were obtained from the animal house of Tabriz University and housed in an animal room in groups of five per cage. They were maintained at $22{ }^{\circ} \mathrm{C}$ with free access to commercial rat chow and tap water. The animals were adapted to an inverse 12/12-hour light/dark cycle for 2 weeks prior to the exercise program. All experimental procedures employed as well as rat care and handling were approved by the Animal Care Committee of the Tabriz University of Medical Sciences and were in accordance with Experimental Animal Laboratory.

\section{Induction of diabetes}

Diabetes was induced in rats by intraperitoneal injection of $60 \mathrm{mg} / \mathrm{kg}$ streptozotocin (STZ) (Sigma, St Louis, MO) (16). Rats in the control groups received an intraperitoneal injection of an equal volume of citrate buffer instead of STZ. Blood samples were obtained from the tail vein and blood glucose concentrations were measured 48 hours after STZ administration and then confirmed by histopathological studies $(17,18)$.

\section{Exercise method}

Thirty rats were randomly allocated into three main groups ( $\mathrm{n}=10$ each): healthy sedentary control (HS), diabetic sedentary control (DS) and diabetic exercise-trained group (DE15) which was subjected to running exercise for 15 days. The exercise protocol was chosen considering the capability to control the intensity

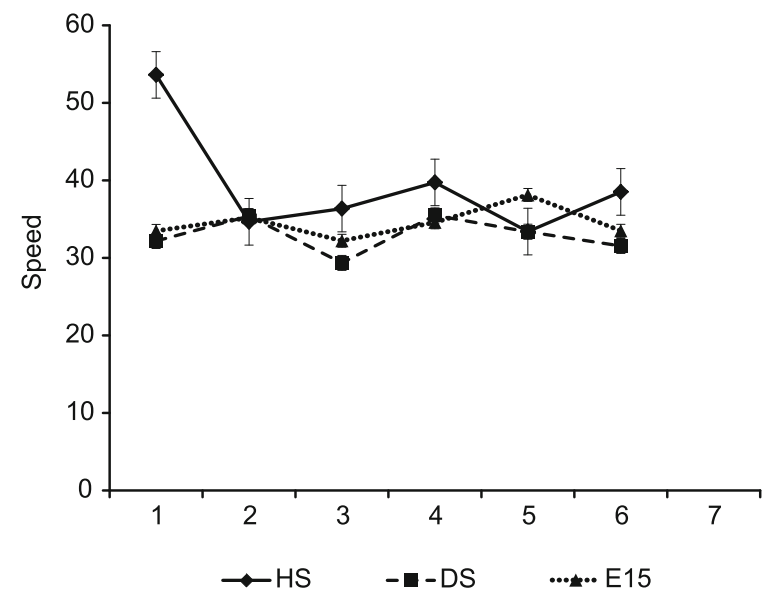

Fig. 1. Average swimming speed. Comparison of average swimming speed between healthy sedentary group (HS), diabetic sedentary group (DS) and diabetic exercise-trained group (DE15). There was no significant difference among different groups. All data were expressed as mean $\pm \mathrm{SE}$.

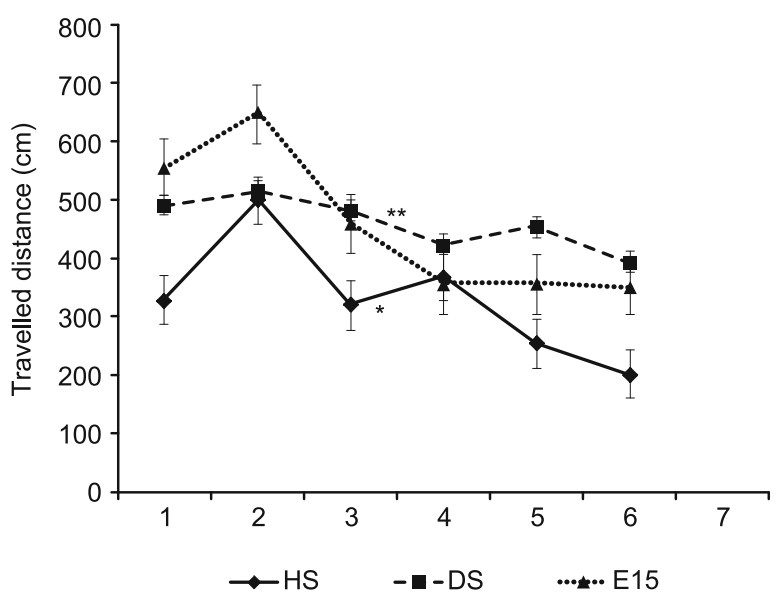

Fig. 2. Average total path. Comparison of average total path between healthy sedentary group (HS), diabetic sedentary group (DS) and diabetic exercise trained group (DE15). There was a significant difference between the healthy sedentary group (HS) and diabetic sedentary group (DS) $(* p<0.05)$. There was a significant difference between the healthy sedentary group (HS) and diabetic exercise-trained group (DE15) $(* * \mathrm{p}<0.05)$. All data were expressed as mean $\pm \mathrm{SE}$.

of exercise, its duration, and timing. All rats in the trained group performed five exercise sessions per duration of $60 \mathrm{~min}$ at a 22 $\mathrm{m} / \mathrm{min}$ pace on a $0 \%$ slope, weekly. The first and last 5 minutes on the treadmill were considered as warm up and recovery periods. One week prior to the beginning of the training protocol, the rats were familiarized with treadmills in order to reduce the environmental stress.

\section{Water maze method (MWM)}

Rats in all study groups were trained on MWM with four trials per day for 6 days. The platform was hidden $2 \mathrm{~cm}$ below the surface of water. The time to reach the platform (latency), distance and speed were calculated (19).

\section{ELISA assay}

ELISA kit Reelin (E92775Ra purchased from Life Science Inc.) was used to measure the levels of the reelin protein according to the kit instruction

\section{Results}

The comparison of swimming speed among groups revealed no significant difference (Fig. 1). The path distance (path length) in the diabetic sedentary group did not show any significant difference in various days. However, a significant difference was observed between the healthy sedentary group (HS) and the diabetic sedentary group (DS) on days 3 and 6 . In addition, there was a significant difference between the healthy sedentary group (HS) and diabetic exercise-trained group (DE15) on day 3 (Fig. 2).

During different days of observation, the rats in DS groups had no significant difference in terms of delayed detection of the platform (escape latency). The escape latency was significantly 


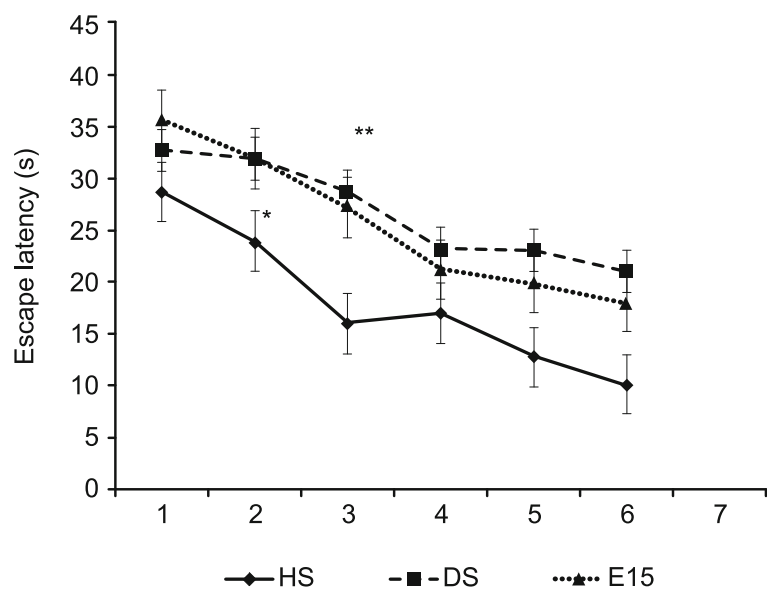

Fig. 3. Average total time. Comparison of average total time between healthy sedentary group (HS), diabetic sedentary group (DS) and diabetic exercise-trained group (DE15). There was a significant difference between the healthy sedentary group (HS) and diabetic sedentary group (DS) $(* p<0.05)$. There was a significant difference between the diabetic sedentary group (DS) and diabetic exercise-trained group (DE15) $(* * \mathbf{p}<0.05)$. All data were expressed as mean \pm SE.

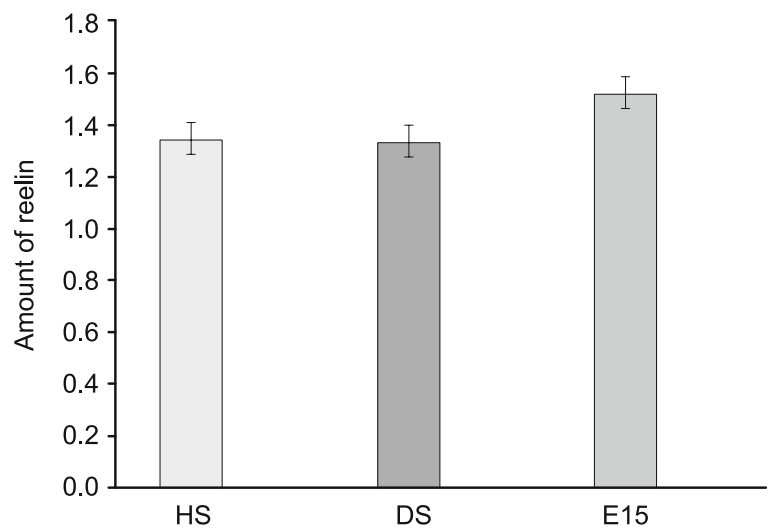

Fig. 4. Reelin levels. Comparison of reelin levels in the hippocampus of rats in healthy sedentary group (HS), diabetic sedentary group (DS) and diabetic exercise trained group (DE15). There was no significant difference between the results of different groups. All data were expressed as mean $\pm \mathrm{SE}$.

different between the healthy sedentary group (HS) and diabetic sedentary group (DS) on day 2. Also, there was a significant difference between the diabetic sedentary control group (DS) and diabetic exercise-trained group (DE15) (Fig. 3).

ELISA test results showed no significant difference in reelin levels between study groups (Fig. 4).

\section{Discussion}

The results of present study showed a significant difference in terms of passed distance and escape latency between the healthy sedentary group (HS) and diabetic sedentary group (DS). Thus, we observed a significantly impaired memory function in the diabetic animals. In this regard, previous investigations have shown that diabetes can cause a wide range of disorders in peripheral and central nervous system (20). Central neuropathy is a common complication of diabetes that is associated with disturbances in learning, memory and cognitive skills (21). These problems emerge over time and increase the risk of dementia and Alzheimer's disease in humans (22). The hippocampus is mostly vulnerable to DM I-induced hyperglycemia (23). Since, it has been considered as the main target of complications subsequent to diabetes, including cognitive dysfunction due to hippocampal neuron damage (24). Although the mechanisms of the damage to hippocampal neurons subsequent to diabetes are unknown, it has been shown that dendritic atrophy, decreased regulation of the glucocorticoid receptor, changes in expression of insulin-like growth factor receptor, reduction in insulin transmitter and induction of apoptosis are major pathologies that occur in neurons (25).

In the present study, the escape latency differed significantly between diabetic sedentary control group (DS) and diabetic exercise-trained group (DE15), which reveals an improvement in spatial memory in diabetic animals through short-term exercise. It should be noted that in the present study, memory was measured after exercise.

Our findings are consistent with previous studies that showed the effect of exercise on the brain and its activity, namely on increase in learning and memory of young rats (26). Similar to our results, it has been reported that treadmill exercise improves learning and memory performance in MWM (27) and proved that short-term treadmill running (for 10 days) increased learning in MWM and elevated the levels of epinephrine (28). In another study, 5 days of voluntary running, increased rats' learning, which was evaluated via MWM (29).

Previous studies summarized that exercise increases long-term potential (LTP) in different regions of the hippocampus and facilitates recovery of brain injury with upregulation of brain-derived neurotrophic factors such as BDNF, which enhances neurogenesis $(30,31)$. BDNF is an essential factor for Cajal Retzius cells located in the hippocampus. Cajal Ratzius cells are also an important source for secretion of reelin (32). It is specified that reelin plays a role in memory and its role has been observed in patients with schizophrenia (31). Stranahan et al. found that reelin plays an important role in the improvement of hippocampus performance (33).

Although, previous studies hypothesized that exercise improves spatial memory in diabetic rats by increasing reelin, our result was inconsistent with literature. Exercise improved spatial memory in diabetic rats but not via reelin increase; it could have been performed through other above-mentioned mechanisms.

\section{References}

1. Burr JF, Shephard RJ, Riddell MC. Physical activity in type 1 diabetes mellitus Assessing risks for physical activity clearance and prescription. Can Fam Physician 2012; 58 (5): 533-535.

2. Ye L, Wang F, Yang RH. Diabetes impairs learning performance and affects the mitochondrial function of hippocampal pyramidal neurons. Brain Res 2011; 1411: 57-64. 
107-110

3. Lobnig BM, Krömeke O, Optenhostert-Porst $\mathrm{C}$ et al. Hippocampal volume and cognitive performance in long-standing Type 1 diabetic patients without macrovascular complications. Diabetic Med 2005; 23 (1): 32-39.

4. Magariños AM, McEwen BS. Experimental diabetes in rats causes hippocampal dendritic and synaptic reorganization and increased glucocorticoid reactivity to stress. Proc Natl Acad Sc 2000; 97 (20): 11056-11061.

5. Martínez-Tellez R, Gómez-Villalobos Mde J, Flores G. Alteration in dendritic morphology of cortical neurons in rats with diabetes mellitus induced by streptozotocin. Brain Res 2005; 1048 (1): 108-115.

6. Borradaile NM, Carroll KK, Kurowska EM. Regulation of HepG2 cell apolipoprotein $\mathrm{B}$ metabolism by the citrus flavanones hesperetin and naringenin. Lipids 1999; 34 (6): 591-598.

7. Wehr HA, Bednarska-Makaruk MA, Lipczynska-Lojkowska W et al. Metabolic syndrome and dementia. Postepy Psychiatri Neurolology 2012; 21: 117-122.

8. Fatemi SH, Earle JA, McMenomy T. Reduction in Reelin immunoreactivity in hippocampus of subjects with schizophrenia, bipolar disorder and major depression. Mol Psychiatry 2000; 5 (6): 654.

9. Beffert U, Weeber EJ, Durudas A et al. Modulation of synaptic plasticity and memory by Reelin involves differential splicing of the lipoprotein receptor Apoer2. Neuron 2005; 47 (4): 567-579.

10. Meyer G, Schaaps JP, Moreau L et al. Embryonic and early fetal development of the human neocortex. J Neurosci 2000; 20 (5): 1858-1868.

11. Fatemi SH, Reutiman TJ, Folsom TD. The role of reelin in etiology and treatment of psychiatric disorders. In Reelin Glycoprotein: Structure, Biology and Roles in Health and Disease. New York: Springer, 2008: 317-339.

12. Abraham H, Meyer G. Reelin-expressing neurons in the postnatal and adult human hippocampal formation. Hippocampus 2003; 13 (6): 715-727.

13. Shapiro LA, Wang L, Upadhyaya P et al. Seizure-induced increased neurogenesis occurs in the dentate gyrus of aged Sprague-Dawley rats. Aging Dis 2011; 2 (4): 286.

14. Riddell M, Perkins BA. Exercise and glucose metabolism in persons with diabetes mellitus: perspectives on the role for continuous glucose monitoring. J Diabetes Sci Technol (Online) 2009; 3 (4): 914.

15. Van Praag H, Kempermann G, Gage FH. Running increases cell proliferation and neurogenesis in the adult mouse dentate gyrus. Nat Neurosci 1999; 2 (3): 266-270.

16. Movahedian A, Zolfaghari B, Sajjadi SE et al. Antihyperlipidemic effect of Peucedanum pastinacifolium extract in streptozotocin-induced diabetic rats. Clinics 2010; 65 (6): 629-633.

17. Nadai M, Yoshizumi H, Kuzuya T et al. Effect of diabetes on disposition and renal handling of cefazolin in rats. Drug Metab Dispos 1990; 18 (5): 565-570.
18. Hoftiezer V, Carpenter A. Comparison of streptozotocin and alloxaninduced diabetes in the rat, including volumetric quantitation of the pancreatic islets. Diabetologia 1973; 9 (3): 178-184.

19. Ayoub RS. Effect of exercise on spatial learning and memory in male diabetic rats. Int J Diabetes Metab 2009; 17: 93-98.

20. Nitta A, Murai R, Suzuki N et al. Diabetic neuropathies in brain are induced by deficiency of BDNF. Neurotoxicol Teratol 2002; 24 (5): 695-701.

21. Roghani M, Joghataie MT, Jalili MR et al. Time course of changes in passive avoidance and Y-maze performance in male diabetic rats. Iranian Biomedical Journal 2006; 10 (2): 99-104.

22. Strachan MW, Price JF, Frier BM. Diabetes, cognitive impairment, and dementia. Br Med J 2008; 336 (7634): 6.

23. Reagan LP, MagariÑos AM, McEwen BS. Neurological changes induced by stress in streptozotocin diabetic rats. Ann N Y Acad Sci 2006; 893 (1): 126-137.

24. Sima AA, Zhang W, Kreipke CW et al. Inflammation in diabetic encephalopathy is prevented by C-peptide. Rev Diabet Stud 2009; 6 (1): 37.

25. Greene D. The pathogenesis and prevention of diabetic neuropathy and nephropathy. Metabolism 1988; 37 (2): 25-29.

26. Uysal N, Tugyan K, Kayatekin BM et al. The effects of regular aerobic exercise in adolescent period on hippocampal neuron density, apoptosis and spatial memory. Neurosci Lett 2005; 383 (3): 241-245.

27. Ang ET, Dawe GS, Wong PT et al. Alterations in spatial learning and memory after forced exercise. Brain Res 2006; 1113 (1): 186-193.

28. Ahmadiasl N, Alaei H, Hanninen O. Effect of exercise on learning, memory and levels of epinephrine in rats hippocampus. J Sports Sci Med 2003; 2 (3): 160-169.

29. Akhavan MM, Emami-Abarghoie M, Sadighi-Moghaddam B et al. Hippocampal angiotensin II receptors play an important role in mediating the effect of voluntary exercise on learning and memory in rat. Brain Res 2008; 1232: 132-138.

30. Toldy A, Stadler K, Sasvári M et al. The effect of exercise and nettle supplementation on oxidative stress markers in the rat brain. Brain Res Bull 2005; 65 (6): 487-493.

31. Wedenoja J, Loukola A, Tuulio-Henriksson A et al. Replication of linkage on chromosome 7q22 and association of the regional Reelin gene with working memory in schizophrenia families. Mol Psychiatry 2007; 13 (7): 673-684.

32. Guidotti A, Auta J, Davis JM et al. Decrease in reelin and glutamic acid decarboxylase67 (GAD67) expression in schizophrenia and bipolar disorder: a postmortem brain study. Arch Gen Psychiatry 2000; 57 (11): 1061-1069.

33. Stranahan AM, Salas-Vega S, Jiam NT et al. Interference with reelin signaling in the lateral entorhinal cortex impairs spatial memory. Neurobiol Learn Mem 2011; 96 (2): 150-155. 